\title{
Retraction Note: miR-663 attenuates tumor growth and invasiveness by targeting eEF1A2 in pancreatic cancer
}

Wenqiao Zang ${ }^{1}$, Yuanyuan Wang ${ }^{1}$, Tao Wang ${ }^{2}$, Yuwen Du', Xiaonan Chen ${ }^{1}$, Min Li ${ }^{1}$ and Guogiang Zhao ${ }^{*}$

\section{Retraction note to: Molecular Cancer}

https://doi.org/10.1186/s12943-015-0315-3

The Editor-in-Chief has retracted this article [1] because Figure $6 \mathrm{~b}$ overlaps with Figure 8 of [2] and Figure 4a overlaps with Figure $2 \mathrm{~b}$ of [3]. An investigation by Zhengzhou University has confirmed that these figures overlap. The data reported in this article are therefore unreliable. Guoqiang Zhao agrees with this retraction. Wenqiao Zang, Yuanyuan Wang, Tao Wang, Yuwen Du, Xiaonan Chen and Min Li have not responded to any correspondence about this retraction.

\section{Author details}

${ }^{1}$ College of Basic Medical Sciences, Zhengzhou University, Zhengzhou 450001, Henan Province, China. ${ }^{2}$ Department of Hemato-Tumor, The First Affiliated Hospital of Henan University of TCM, Zhengzhou 450000, China.

Published online: 18 July 2019

\section{References}

1. Zang W, Wang Y, Wang T, Du Y, Chen X, Li M, et al. miR-663 attenuates tumor growth and invasiveness by targeting eEF1A2 in pancreatic cancer. Mol Cancer. 2015;14:37. https://doi.org/10.1186/s12943-015-0315-3.

2. Wang T, Zang W, Li M, et al. Dig Dis Sci. 2013;58:706. https://doi.org/10. 1007/s10620-012-2395-x.

3. Wang T, Xuan X, Li M, et al. Diagn Pathol. 2013;8:179. https://doi.org/10. 1186/1746-1596-8-179 Retracted article.

\footnotetext{
* Correspondence: zhaogq@zzu.edu.cn

${ }^{1}$ College of Basic Medical Sciences, Zhengzhou University, Zhengzhou

450001, Henan Province, China

Full list of author information is available at the end of the article
} 